\title{
Strict uniformization of real algebraic curves and global real analytic coordinates on real Teichmüller spaces.
}

\author{
J. HUISMAN
}

\begin{abstract}
We construct a global system of real analytic coordinates on the real Teichmüller space of a compact real algebraic curve $X$, using so-called strict uniformization of the real algebraic curve $X$. A global coordinate system is then obtained via real quasiconformal deformations of the Kleinian subgroup of $\mathrm{PGL}_{2}(R)$ obtained as a group of covering transformations of a strict uniformization of $X$.
\end{abstract}

\section{Introduction}

The object of this paper is to construct a global system of real analytic coordinates on the real Teichmüller space of a compact real algebraic curve.

In the litterature one can find several global systems of complex analytic coordinates on the complex Teichmüller space $T(X)$ of a compact complex algebraic curve $X[3,5,8,9,13]$. If the complex algebraic curve $X$ can be defined be polynomial equations with coefficients in $\boldsymbol{R}$, then the Galois group $\Sigma$ of $\mathbb{C}$ over $\boldsymbol{R}$ acts naturally on $T(X)$. Some of the fore-mentioned coordinate systems then, are equivariant with respect to the $\Sigma$-action on $T(X)[3,5,8,9]$ and hence, induce global systems of real analytic coordinates on the real Teichmüller space $T(X)^{\Sigma}$ of the real algebraic curve $X$. These coordinate systems are relatively complicated. For example, it seems not feasible to determine explicitly their imagewhich would have its interest in questions concerning moduli spaces of

1991 Mathematics Subject Classification: 32G15, 14P99, $14 \mathrm{H} 30$.

Servicio Publicaciones Univ. Complutense. Madrid, 1999. 
real algebraic curves.

One can hope to find a more simple coordinate system on $T(X)^{\Sigma}$ by constructing a global system of real analytic coordinates on $T(X)^{\Sigma}$ which does not necessarily extend to a global system of complex analytic coordinates on $T(X)$. This is indeed the case, as we will show in the present paper.

The idea of construction is roughly the following. Given a compact real algebraic curve $X$ of genus $g \geq 2$, there is a uniformization $p: \Omega \rightarrow X$ of $X$ by an open subset $\Omega$ of the Riemann sphere $P^{1}(\mathscr{C})$ having the following properties.

1. The map $p$ is a holomorphic covering map.

2. The set $\Omega$ is stable for the action of the Galois group $\Sigma$ of $\mathbb{C}$ over $\mathbb{R}$ and the map $p$ is equivariant with respect to the action of $\Sigma$.

3. The inverse image $p^{-1}\left(X^{\Sigma}\right)$ of the set of real points $X^{\Sigma}$ of $X$ is equal to $\Omega \cap P^{1}(R)$

In fact, $p$ is universal among all uniformizations of $X$ having the above properties. Such a uniformization will be called a strict uniformization of $X$. Such a uniformization of a real algebraic curve seems to have been considered for the first time in :

Koebe, P.: Über die Uniformisierung reeller algebraischer Kurven. Nachr. Akad. Wiss. Göttingen (1907), 177-190.

Let $G$ be the group of automorphisms of the covering $p$ of $X$ which are equivariant with respect to the action of $\Sigma$ on $\Omega$. Then, $G$ turns out to be a Kleinian subgroup of $\mathrm{PGL}_{2}(\mathbb{R})$ and its quasiconformal deformations in $\mathrm{PGL}_{2}(\boldsymbol{R})$ turn out to parametrize the real Teichmüller space of $X$.

The group $G$ is what we will call the strict fundamental group of $X$ and has a particularly easy presentation. If $X$ does have real points, then $G$ is a free group on $g$ generators. If $X$ does not have real points, then $G$ is a group on $g+1$ generators satisfying a simple relation. In both cases, one can easily construct a global system of real analytic coordinates on the real quasiconformal deformation space of $G$. That coordinate system will then give rise to a global system of real analytic coordinates on the real Teichmüller space of $X$. The image of the latter coordinate system will be studied in a forthcoming paper [10]. 
The paper is organized as follows. Section 2 introduces briefly the equivariant theory of topological covering maps and introduces the strict fundamental group of a transformation space. Section 3 relates so-called strict equivariant coverings of a transformation space to ordinary coverings of its quotient. Section 4 determines the strict fundamental group of a compact real algebraic curve. Section 5 recalls what uniformization of a real algebraic curve by the double half-plane is. That is then used in Section 6 to prove strict uniformization of compact real algebraic curves. All this is then used in Section 7 to construct a global system of real analytic coordinates on the real Teichmüller space of a real algebraic curve.

Convention. A Riemann surface is not necessarily connected nor compact.

\section{The universal strict equivariant covering}

Let $\Sigma$ be any group. A $\Sigma$-space is a topological space $X$ endowed with an action of $\Sigma$ such that every element of $\Sigma$ acts continuously on $X$. A subset $U$ of $X$ is said to be stable if $\sigma \cdot u \in U$ for all $u \in U$ and all $\sigma \in \Sigma$. A $\Sigma$-space $X$ is said to be equivariantly connected if $\emptyset$ and $X$ are the only open and closed stable subsets of $X$. If $X$ is locally connected, then $X$ is equivariantly connected if and only if $\Sigma$ acts transitively on the set of connected components of $X$.

A continuous map $f: Y \rightarrow X$ of $\Sigma$-spaces is sajd to be equivariant if $f(\sigma \cdot y)=\sigma \cdot f(y)$ for all $y \in Y$ and all $\sigma \in \Sigma$. An equivariant base point of a $\Sigma$-space $X$ is an equivariant map $b: \Sigma \rightarrow X$. Let $X$ and $Y$ be $\Sigma$-spaces and let $b$ and $c$ be equivariant base points of $X$ and $Y$, respectively. An equivariant map $f: Y \rightarrow X$ is said to be equivariant base point-preserving if $f \circ c=b$. We denote this by $f:(Y, c) \rightarrow(X, b)$.

An equivariant covering of a $\Sigma$-space $X$ is an equivariant map $p: Y \rightarrow$ $X$ of $\Sigma$-spaces which is a covering map. Recall that a morphism from a covering $p: Y \rightarrow X$ into a covering $q: Z \rightarrow X$ is a continuous map $f: Y \rightarrow Z$ such that $q \circ f=p$. A morphism of equivariant coverings is a morphism of coverings that is equivariant.

An equivariant covering $p:(\hat{X}, \hat{b}) \rightarrow(X, b)$, where $\hat{b}$ and $b$ are equivariant base points, is said to be a universal equivariant covering of $X$ if, for 
every covering $q:(Y, c) \rightarrow(X, b)$, there is a unique morphism of equivariant coverings $f:(\hat{X}, \hat{b}) \rightarrow(Y, c)$ such that $q \circ f=p$.

Let $X$ be a $\Sigma$-space, and let $b$ be an equivariant base point of $X$. Suppose that $X$ has a universal equivariant covering $p:(\hat{X}, \hat{b}) \rightarrow(X, b)$. By the universal property of such a covering, the group Aut $(\hat{X} / X)$ is uniquely determined by $X$, up to unique isomorphism.

Definition 2.1. The group Aut $(\hat{\mathrm{X}} / \mathrm{X})$ of automorphisms of the equivariant covering $\hat{X}$ of $X$ is called the equivariant fundamental group of $X$, and is denoted by $\pi_{1}(X, \Sigma ; b)$, or simply by $\pi_{1}(X, \Sigma)$.

The following proposition gives a criterion for a universal equivariant covering to exist. For a proof, one is referred to [8].

Proposition 2.2. Let $X$ be a locally and equivariantly connected $\Sigma$ space. Let $b$ be an equivariant base point of $X$. Let $X_{\sigma}$ be the connected component of $X$ containing $b_{\sigma}$ for all $\sigma \in \Sigma$. Then, the following conditions are equivalent.

1. The $\Sigma$-space $X$ has a universal equivariant covering.

2. The topological space $X_{\sigma}$ has a universal covering for all $\sigma \in \Sigma$.

Moreover, if one of these conditions is satisfied, a universal equivariant covering $p:(\hat{X}, \hat{b}) \longrightarrow(X, b)$ is Galois; i.e., the map $p$ induces a homeomorphism

$$
\hat{X} / \pi_{1}(X, \Sigma) \cong X
$$

An equivariant map $f: Y \rightarrow X$ of $\Sigma$-spaces is said to be strict if $f$ maps any $\Sigma$-orbit in $Y$ bijectively onto a $\Sigma$-orbit in $X$. The map $f$ is a strict equivariant covering if $f$ is an equivariant covering map that is strict. A morphism of strict equivariant coverings is a morphism of equivariant coverings. Note that such a morphism is automatically strict.

A strict equivariant covering $p:(\tilde{X}, \tilde{b}) \rightarrow(X, b)$, where $\tilde{b}$ and $b$ are equivariant base points, is said to be universal strict equivariant covering of $X$ if, for every strict covering $q:(Y, c) \rightarrow(X, b)$, there is a unique morphism of strict equivariant coverings $f:(\tilde{X}, \tilde{b}) \rightarrow(Y, c)$ such that $q \circ f=p$. 
Let $X$ be a $\Sigma$-space, and let $b$ be an equivariant base point of $X$. Suppose that $X$ has a universal strict equivariant covering $p:(\tilde{X}, \tilde{b}) \rightarrow$ $(X, b)$. By the universal property of such a covering, the group Aut $(\tilde{\mathrm{X}} / \mathrm{X})$ is uniquely determined by $X$, up to unique isomorphism.

Definition 2.3. The group Aut $(\tilde{\mathrm{X}} / \mathrm{X})$ of automorphisms of the strict equivariant covering $\tilde{X}$ of $X$ is called the strict fundamental group of $X$, and will be denoted by $\sigma_{1}(X, \Sigma ; b)$, or simply by $\sigma_{1}(X, \Sigma)$.

We will deduce from the following lemma a criterion for a universal strict equivariant covering to exist.

Lemma 2.4. Let $X$ and $Y$ be locally connected $\Sigma$-spaces. Let $p: Y \rightarrow X$ be an equivariant covering. Then, there is a strict equivariant covering $\tilde{p}: \tilde{Y} \rightarrow X$, and a morphism of equivariant coverings $f: Y \rightarrow \tilde{Y}$ having the following universal property. For any strict equivariant covering $q: Z \rightarrow X$, and any morphism of equivariant coverings $g: Y \rightarrow Z$, there is a unique morphism of strict equivariant coverings $\tilde{g}: \dot{Y} \rightarrow Z$ making the following diagram commutative.

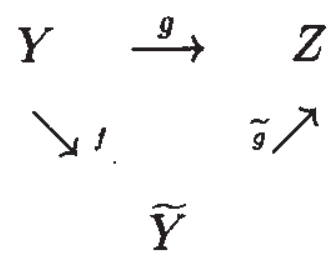

Proof. Let $S$ be the subset of the fiber product $Y \times_{X} Y$ consisting of all pairs $(x, y)$ such that $x$ and $y$ belong to the same $\Sigma$-orbit in $Y$. Let $R$ be the smallest open and closed subset of $Y \times_{X} Y$ containing $S$ and which is an equivalence relation on $Y$. Observe that such a subset exists since $Y \times{ }_{X} Y$ is locally connected. Let $\tilde{Y}$ be the quotient $Y / R$. Let $f$ be the quotient map from $Y$ onto $\tilde{Y}$. Since the equivalence relation $R$ is contained in $Y \times_{X} Y$, the map $p$ factorizes through $f$, i.e., there is a continuous map $\tilde{p}$ from $\tilde{Y}$ onto $X$ such that $\tilde{p} \circ f=p$. Since $R$ is an open and closed subset of $Y \times_{X} Y$, the map $\tilde{p}$ is a covering map.

Consider the natural action of $\Sigma$ on $Y \times_{X} Y$. Obviously, $\sigma \cdot S=S$ for all $\sigma \in \Sigma$. Hence, $\sigma \cdot R$ also is an equivalence relation on $Y$, open and closed in $Y \times_{X} Y$ and containing the subset $S$. Since $R$ is the smallest equivalence relation having this property, one has $\sigma \cdot R \subseteq R$ for all $\sigma \in \Sigma$. 
It follows that the action of $\Sigma$ on $Y$ induces an action of $\Sigma$ on $\tilde{Y}$ and the maps $f$ and $\tilde{p}$ are equivariant.

Since the equivalent relation $R$ on $Y$ contains the set $S$, the equivariant covering map $\tilde{p}$ is strict. We show that this strict equivariant covering of $X$ satisfies the required universal property.

Let $q: Z \rightarrow X$ be a strict equivariant covering of $X$ and let $g: Y \rightarrow Z$ be a morphism of equivariant coverings of $X$. Consider the equivalence relation $R^{\prime}=Y \times{ }_{Z} Y$ on $Y$. Since $q \circ g=p$, the set $R^{\prime}$ is a subset of $Y \times_{X} Y$. Since the covering $q$ is strict, $S \subseteq R^{\prime}$. Moreover, $R^{\prime}$ is open and closed in $Y \times_{X} Y$ since the map $g$ is a covering map of $Z$. It follows that $R$ is contained in $R^{\prime}$ and, hence, that there is a unique continuous map $\tilde{g}$ from $\tilde{Y}$ into $Z$ such that $\tilde{g} \circ f=g$. It is clear that $\tilde{g}$ is equivariant.

Proposition 2.5. Let $X$ be a locally and equivariantly connected $\Sigma$ space, and let $b$ be an equivariant base point of $X$. Let $X_{\sigma}$ be the connected component of $X$ containing $b_{\sigma}$ for all $\sigma \in \Sigma$. Suppose that the topological space $X_{\sigma}$ has a universal covering for all $\sigma \in \Sigma$. Then, the $\Sigma$ space $X$ has a universal strict equivariant covering $\tilde{p}:(\tilde{X}, \tilde{b}) \longrightarrow(X, b)$. Moreover, this universal strict equivariant covering is Galois; i.e., $\tilde{p}$ induces a homeomorphism

$$
\tilde{X} / \sigma_{1}(X, \Sigma) \cong X
$$

Proof. By Proposition 2.2 there is a universal equivariant covering $p:(\hat{X}, \hat{b}) \rightarrow(X, b)$ of $X$. It follows readily from Lemma 2.4 that the induced strict equivariant covering

$$
\tilde{p}:(\tilde{X}, \tilde{b}) \longrightarrow(X, b)
$$

is universal. It also follows from Lemma 2.4 that the the action of $\pi_{1}(X, \Sigma)$ on $\hat{X}$ induces an action of $\pi_{1}(X, \Sigma)$ on $\tilde{X}$. Since the latter action is one acting by automorphisms of the strict equivariant covering $\tilde{p}$, one has induced surjective maps of the quotients

$$
\hat{X} / \pi_{1}(X, \Sigma) \longrightarrow \tilde{X} / \pi_{1}(X, \Sigma) \longrightarrow \tilde{X} / \sigma_{1}(X, \Sigma) \longrightarrow X .
$$

According to Proposition 2.2, the composition of these maps is a homeomorphism. It follows that each of the above maps is a homeomorphism. In particular, $\tilde{p}$ induces a homeomorphism from $\tilde{X} / \sigma_{1}(X, \Sigma)$ onto $X$. 


\section{Universal coverings and universal strict equivariant coverings}

It will be useful to relate strict coverings of a $\Sigma$-space $X$ to ordinary coverings of the quotient space $X / \Sigma$. First, we need to introduce some notation.

Let $X$ be a $\Sigma$-space. Denote by SCovx the category of strict equivariant coverings of $X$ and denote by $\operatorname{Cov}_{X / \Sigma}$ the category of coverings of the topological space $X / \Sigma$. One has a functor

$$
F: \operatorname{Cov}_{\mathrm{x} / \Sigma} \longrightarrow \mathrm{SCovx} .
$$

Indeed, let $\pi: X \rightarrow X / \Sigma$ be the quotient map. If $f: Y \rightarrow X / \Sigma$ is a covering, the fiber product $Y \times_{X / \Sigma} X$ is a topological space on which $\Sigma$ acts diagonally. The projection on the second factor from $Y \times_{X / \Sigma} X$ onto $X$ is an equivariant covering of $X$. We denote this map by $f_{X}$. It is trivial to check that $f_{X}$ is a strict covering of $X$. Define the functor $F$ on objects as $F(f)=f_{X}$. It is clear what the functor $F$ should be on morphisms.

Recall that an action of a group $\Sigma$ on a topological space $X$ is said to be discontinuous if for all $x \in X$, there is an open neighborhood $U$ of $x$ such that

1. $(\sigma \cdot U) \subseteq U$ for all $\sigma \in \Sigma_{x}$, and

2. $(\sigma \cdot U) \cap U=\emptyset$ for all $\sigma \in \Sigma \backslash \Sigma_{x}$,

where $\Sigma_{x}$ denotes the stabilizer of $x$.

Proposition 3.1. Let $X$ be a locally connected $\Sigma$-space. Suppose that $\Sigma$ acts discontinuously on $X$. Then, the functor $F: \operatorname{Cov}_{X / \Sigma} \longrightarrow$ SCovx is an equivalence of categories.

Proof. We define a functor

$$
G: \operatorname{SCovx}_{\mathrm{x}} \longrightarrow \operatorname{Cov}_{\mathrm{x} / \mathrm{\Sigma}}
$$

as follows. Let $g: Z \rightarrow X$ be a strict covering of $X$. One has an induced map $f: Z / \Sigma \rightarrow X / \Sigma$ and one needs to show that $f$ is a covering of $X / \Sigma$. It will then follow that $G(g)=f$ defines a functor from SCovx into Cov $x / \Sigma$. 
Let $x$ be any element of $X$. Let $U$ be an open neighborhood of such that $(\sigma \cdot U) \subseteq U$ for all $\sigma \in \Sigma_{x}$, and $(\sigma \cdot U) \cap U=\emptyset$ for all $\sigma \in \Sigma \backslash \Sigma_{x}$. Since $g$ is a covering, the inverse image $g^{-1}(U)$ of $U$ is a disjoint union $\bigsqcup_{i \in I} V_{i}$ of open subsets $V_{i}$ of $Z$. Moreover, the restriction of $g$ to each $V_{i}$ is a homeomorphism onto $U$.

Since $U$ is stable for the action of $\Sigma_{x}$, the inverse image $g^{-1}(U)$ is also stable for the action of $\Sigma_{x}$. The topological space $X$ being locally connected, we may assume $U$ to be connected. Then, each $V_{i}$ is connected. Let $y_{i}$ be the inverse image of $x$ in $V_{i}$. Since the covering $g$ is strict, $y_{i}$ is a fixed point of $\Sigma_{x}$. By connectedness of $V_{i}$, the open subset $V_{i}$ of $Z$ is $\Sigma_{x^{-}}$-stable.

Since $(\sigma \cdot U) \cap U=\emptyset$ for all $\sigma \in \Sigma \backslash \Sigma_{x}$, the quotient $U / \Sigma_{x}$ is an open neighborhood of $\pi(x)$ in $X / \Sigma$. Similarly, $g^{-1}(U) / \Sigma_{x}$ can be considered as an open subset of $Z / \Sigma$. The inverse image of $U / \Sigma_{x}$ by $f$ is equal to this open subset of $Z / \Sigma$. The quotient $g^{-1}(U) / \Sigma_{x}$ is equal to the disjoint union of the quotients $V_{i} / \Sigma_{x}$, each of which is mapped homeomorphically by $f$ onto $U / \Sigma_{x}$. Therefore, $f$ is a covering map.

It is easy to check that the functors $F$ and $G$ are quasi-inverses of each other.

Corollary 3.2. Let $X$ be a locally and equivariantly connected $\Sigma$-space. Suppose that $\Sigma$ acts discontinuously on $X$. Let $b$ be an equivariant base point of $X$. Let

$$
\tilde{p}:(\tilde{X}, \tilde{b}) \rightarrow(X, b)
$$

be a strict equivariant covering of $X$. Denote by

$$
p:(\tilde{X} / \Sigma, \overline{\tilde{b}}) \rightarrow(X / \Sigma, \bar{b})
$$

the induced ondinary covering of the quotient space $X / \Sigma$, where $\overline{\tilde{b}}$ and $\vec{b}$ denote the induced ondinary base points of $\tilde{X} / \Sigma$ and $X / \Sigma$, respectively. Then, $\tilde{p}$ is a universal strict equivariant covering if and only if $p$ is a universal covering. In particular, the following conditions are equivalent.

1. The $\Sigma$-space $X$ has a universal strict equivariant covering.

2. The quotient space $X / \Sigma$ has a universal covering. 
Moreover, if one of these conditions is satisfied then one has an isomorphism

$$
\sigma_{1}(X, \Sigma) \cong \pi_{1}(X / \Sigma)
$$

of fundamental groups.

Remark 3.3. Observe that Propositions 2.2 and 2.5 and Corollary 3.2 show the following statement. Let $X$ be a locally and equivariantly connected $\Sigma$-space. Suppose that $\Sigma$ acts discontinuously on $X$. If all connected components of $X$ admit a universal covering, then the quotient $X / \Sigma$ admits a universal covering.

\section{The strict fundamental group of real alge- braic curves}

Let $\Sigma$ be the Galois group of $\boldsymbol{C}$ over $\boldsymbol{R}$, i.e., $\Sigma=\{1, \sigma\}$, where $\sigma$ is complex conjugation. Let $X$ be a Riemann surface. A real structure on $X$ is an action of $\Sigma$ on $X$ such that $\sigma$ acts antiholomorphically. We will also say that such a Riemann surface is defined over $\boldsymbol{R}$. We denote by $X^{\Sigma}$ the subset of fixed points for the action on $X$ by $\Sigma$. The points of $X^{\Sigma}$ are called real points of $X$.

Recall that a Riemann surface $X$ is of finite type if $X$ is biholomorphic to the complement of a finite set in a compact Riemann surface. A Riemann surface of finite type is essentially a complex algebraic curve. Similarly, a Riemann surface $\boldsymbol{X}$ defined over $\boldsymbol{R}$ which is of finite type is essentially a real algebraic curve. Therefore, in what follows, by a complex algebraic curve (resp. a real algebraic curve) we mean a Riemann surface of finite type (resp. a Riemann surface of finite type defined over $\boldsymbol{R}$ ).

Let $X$ be a compact connected real algebraic curve. Let $g=g(X)$ be the genus of $X$. The number of connected components of $X^{\Sigma}$ will be denoted by $s=s(X)$. The real algebraic curve is said to be dividing if $X \backslash X^{\Sigma}$ is not connected. It is well known that $s \equiv g+1 \bmod 2$ and $1 \leq s \leq g+1$ if $X$ is dividing, and that $0 \leq s \leq g$ if $X$ is nondividing.

Proposition 4.1. Let $X$ be a compact connected real algebraic curve. Let $g=g(X)$ and $s=s(X)$. Then, the strict equivariant fundamental group $\sigma_{1}(X, \Sigma)$ is isomorphic to the group generated by elements $\gamma_{1}, \ldots, \gamma_{g+1}$ subject to the following relation. 
1. If $X$ is dividing then

$$
\gamma_{1} \cdots \gamma_{s} \cdot\left[\gamma_{s+1}, \gamma_{s+2}\right] \cdots\left[\gamma_{g}, \gamma_{g+1}\right]=1
$$

2. If $X$ is nondividing then

$$
\gamma_{1} \cdots \gamma_{s} \cdot \gamma_{s+1}^{2} \cdots \gamma_{g+1}^{2}=1
$$

In particular, the group $\sigma_{1}(X, \Sigma)$ is a free group on generators if $X^{\Sigma} \neq$ 0. The group $\sigma_{1}(X, \Sigma)$ is isomorphic to the group

$$
<\gamma_{1}, \ldots, \gamma_{g+1} \mid \gamma_{1}^{2} \cdots \gamma_{g+1}^{2}>
$$

if $X^{\Sigma}=\emptyset$.

Proof. Since the Euler characteristic $\chi\left(X^{\Sigma}\right)$ of the set of fixed points $X^{\Sigma}$ of $X$ is equal to 0 , one has $\chi(X / \Sigma)=\frac{1}{2} \chi(X)=1-g$. Let $S$ be a connected compact surface such that $X / \Sigma$ is homeomorphic to the complement of the union of $s$ disjoint open discs in $S$. Then, $\chi(S)=$ $1-g+s$

If $X$ is dividing then $X / \Sigma$ is orientable. The same then holds for $S$. It follows that $S$ is an orientable surface of genus $\frac{1}{2}(g-s+1)$. Then, the group $\sigma_{1}(X, \Sigma)$, being isomorphic to the fundamental group of $X / \Sigma$ by Corollary 3.2, is generated by elements $\gamma_{1}, \ldots, \gamma_{g+1}$, subject to relation 1 .

If $X$ is nondividing then $X / \Sigma$ is nonorientable, and so is $S$. It follows that $S$ is the connected sum of $g+1-s$ real projective planes. In this case, the group $\sigma_{1}(X, \Sigma)$ is then generated by elements $\gamma_{1}, \ldots, \gamma_{g+1}$ subject to relation 2 .

\section{Uniformization of real algebraic curves}

For the convenience of the reader we recall some facts on ordinary uniformization of real algebraic curves before discussing their strict uniformization. For proofs, the reader is refered to [8].

We will call a connected Riemann surface hyperbolic if it is universally covered, in the holomorphic sense, by the upper half-plane $\mathbb{U}$. An 
equivariantly connected Riemann surface defined over $\boldsymbol{R}$ will be said to be hyperbolic if each of its connected components is a hyperbolic Riemann surface.

We denote the upper (resp. lower) half-plane by (resp. L), and we denote by $\boldsymbol{D}$ the double half-plane $\boldsymbol{U} \cup \mathrm{L}$. The uniformization of Riemann surfaces over $\boldsymbol{R}$ is then merely a consequence of the classical uniformization of Riemann surfaces ([6], Theorem IV.4.1).

Theorem (Uniformization of Riemann surfaces over $\mathbb{R}$ ).

Let $X$ be a hyperbolic equivariantly connected Riemann surface defined over $\boldsymbol{R}$. Then, there is a universal equivariant holomorphic covering $p: D \rightarrow X$ of $X$ by the double half-plane $\boldsymbol{D}$.

A universal equivariant holomorphic covering $p: D \rightarrow X$ will be called a uniformization of the Riemann surface $X$ over $\boldsymbol{R}$. In case $X$ is a real algebraic curve, a uniformization of $X$ as a Riemann surface defined over $\boldsymbol{R}$ will be called a uniformization of $X$ as a real algebraic curve.

If $p: \mathbb{D} \rightarrow X$ is a uniformization of a Riemann surface $X$ over $\mathbb{R}$, then the group $G$ of automorphisms of $p$ acts holomorphically on $D$, i.e., $G$ is a subgroup of the group $\operatorname{Aut}_{\Sigma}(D)$ of equivariant automorphisms of $\boldsymbol{D}$. The group $\operatorname{Aut}_{\Sigma}(\boldsymbol{D})$ is nothing but the group $\mathrm{PGL}_{2}(\boldsymbol{R})$ acting on $D$ by Möbius transformations. Hence, the group $G$ is a subgroup of $\mathrm{PGL}_{2}(\boldsymbol{R})$. Since $G$ acts discontinuously on $\boldsymbol{D}$, the group $G$ is Kleinian. (We refer to [14] for definitions and facts concerning Kleinian groups.)

We will say that a Kleinian subgroup $G$ of $\mathrm{PGL}_{2}(R)$ is of the first $k i n d$ if its region of discontinuity is equal to $D$. Otherwise, $G$ is said to be of the second kind. In that case, the domain of discontinuity of $G$ contains $D$ as a proper subset, and the limit set of $G$ is a nowhere dense subset of $P^{1}(R)$. Note that the definition of the kind of a Kleinian subgroup of $\mathrm{PGL}_{2}(\boldsymbol{R})$ extends the classical definition in case $G$ is contained in $\operatorname{PSL}_{2}(R)$, i.e., in case $G$ is Fuchsian.

Proposition 5.1. Let $X$ be a hyperbolic equivariantly connected Riemann surface defined over $\boldsymbol{R}$. Let $p: D \rightarrow X$ be a universal equivariant holomorphic covering of $X$. Let $G$ be the group of automorphisms of the covering $p$. Then,

1. the group $G$ is isomorphic to the equivariant fundamental group $\pi_{1}(X, \Sigma)$ of $X$ 
2. the group $G$ is a Kleinian subgroup of $\mathrm{PGL}_{2}(\mathbb{R})$, acting discontinuously on $D$;

3. the quotient Riemann surface $D / G$ is equivariantly isomorphic to $X$.

Moreover, the following equivalences hold.

4. The group $G$ is of the second kind if and only if the Riemann surface $X$ has a nonempty ideal boundary.

5. The group $G$ is Fuchsian if and only if $X$ is not connected.

6. The group $G$ contains parabolic elements if and only if $X$ has punctures.

7. The group $G$ contains elliptic elements if and only if $X$ has real points.

\section{Strict uniformization of real algebraic curves}

In this section we show that, with a few exceptions, a real algebraic curve can be uniformized by an open subset of the Riemann sphere such that this uniformization is, in fact, a universal strict equivariant covering of the real algebraic curve.

Theorem (Strict uniformization of real algebraic curves)

Let $X$ be a compact connected real algebraic curve of genus $g$. Suppose that $X^{\Sigma}$ is nonempty if $g=0$ or 1 . Then, there is a $\Sigma$-stable open subset $\Omega$ of $\boldsymbol{P}^{1}(\mathcal{Q})$ containing the double half-plane $\mathbb{D}$, such that there is a universal strict equivariant holomorphic covering $\tilde{p}: \Omega \rightarrow X$ of $X$ by $\Omega$.

Proof. Let $Y$ be the complement of $X^{\Sigma}$ in $X$. Then, $Y$ is an equivariantly connected Riemann surface defined over $\mathbb{R}$. Observe that $Y$ is hyperbolic. Indeed, if $X^{\Sigma}$ is nonempty then every connected component of $Y$ has a nonempty ideal boundary, hence $Y$ is hyperbolic. If $X^{\Sigma}$ is empty, then by hypothesis, $X$ is of genus greater than or equal to 2 . Then, since $X=Y$ and $X$ is hyperbolic, $Y$ is hyperbolic too. 
Applying to $Y$ uniformization of Riemann surfaces over $\mathbb{R}$, there is a universal equivariant holomorphic covering $p: D \rightarrow Y$ of $Y$ by the double half-plane $\boldsymbol{D}$.

Let $G$ be the group of equivariant automorphisms of $p$. Then, $G$ is a Kleinian subgroup of $\mathrm{PGL}_{2}(\boldsymbol{R})$. Let $\Omega$ be its region of discontinuity. Then, $\Omega$ is stable for the action of $\Sigma$. We show that $G$ acts freely, on $\Omega$. That will allows us to extend $p$ to a map $\vec{p}$ from $\Omega$ into $X$. Then, we will show that $\tilde{p}$ is a universal strict equivariant holomorphic covering of $X$.

Suppose that $G$ acts not freely on $\Omega$. Let $x \in \Omega$ have a nontrivial stabilizer $G_{x}$. Let $\gamma \in G_{x}$ be nontrivial. Then, $\gamma$ is an elliptic Möbius transformation. But $Y$ does not have real points. According to Proposition 5.1, $G$ does not contain eliptic elements. Contradiction, i.e., $G$ acts freely on $\Omega$.

Let $p_{1}$ be the restriction of $p$ to $\mathbb{U}$, and $p_{2}$ the restriction of $p$ to L. Since $p$ is equivariant, $p_{2}(z)=\sigma\left(p_{1}(\sigma(z))\right)$ for all $z \in \mathrm{L}$. Since $p_{1}$ is conformal and $G$ acts freely on all of $\Omega$, the map $p_{1}$ extends uniquely to a continuous map $\tilde{p}_{1}$ from $\Omega \cap \bar{W}$ into $X$ (cf. [2], Satz IV.8.41). Similarly, $p_{2}$ extends uniquely to a continuous map $\tilde{p}_{2}$ from $\Omega \cap \overline{\mathrm{L}}$ into $X$. Then, by uniqueness of $\tilde{p}_{2}$, one has $\tilde{p}_{2}(z)=\sigma \cdot \tilde{p}_{1}(\sigma(z))$ for all $z \in \Omega \cap \overline{\mathrm{L}}$. In particular, $\tilde{p}_{1}$ and $\tilde{p}_{2}$ coincide on $\Omega \cap \boldsymbol{P}^{1}(\mathbb{R})$. Hence, they induce a continuous map $\tilde{p}: \Omega \rightarrow X$ whose restriction to $D$ is equal to $p$. By the so-called analytic definition of quasiconformal mappings ([12], Theorem IV.2.3), $\tilde{p}$ is quasiconformal. Since its restriction to $\boldsymbol{D}$ is holomorphic and $\Omega \backslash \boldsymbol{D}$ is of measure $0, \tilde{p}$ is holomorphic on all of $\Omega$.

It is clear that the elements of $G$ act as equivariant automorphisms of the map $\tilde{p}$. Hence, $\tilde{p}$ induces a holomorphic map $f$ from the Riemann surface $\Omega / G$ into $X$. Its restriction to the open subset $D / G$ is an isomorphism onto its image $Y$. Therefore, $f$ is an open embedding of $\Omega / G$ into $X$.

Since $Y$ has no punctures, the group $G$ is loxodromic by Proposition 5.1. Therefore, the quotient $\Omega / G$ is compact. It follows that $f$ is an isomorphism onto $X$, i.e., the map $\tilde{p}: \Omega \rightarrow X$ is surjective. Since $\Omega / \Sigma$ is simply connected, the induced map $\Omega / \Sigma \rightarrow X / \Sigma$ is a universal covering of $X / \Sigma$. By Corollary $3.2, \tilde{p}$ is a universal strict equivariant covering of $X$. 
A universal strict equivariant holomorphic covering $\tilde{p}: \Omega \rightarrow X$ of a real algebraic curve $X$ will be called a strict uniformization of $X$.

Remark 6.1. Let $p: \Omega \rightarrow X$ be a strict uniformization of a real algebraic curve $X$. By Corollary 3.2, the induced map on the quotients

$$
\tilde{p}: \Omega / \Sigma \longrightarrow X / \Sigma
$$

is a universal dianalytic covering of the Klein surface $X / \Sigma$ associated to $X$ (see [1] for the theory of Klein surfaces and dianalytic maps). The simply connected Klein surface $\Omega / \Sigma$ is open in the Klein surface $\boldsymbol{P}^{1}(\mathcal{Q}) / \Sigma$. Strict uniformization of real algebraic curves thus implies uniformization of Klein surfaces.

Proposition 6.1. Let $X$ be a compact connected real algebraic curve of genus $g$. Suppose that $X^{\Sigma}$ is nonempty if $g=0$ or 1 . Let $\tilde{p}: \Omega \rightarrow X$ be a universal strict equivariant holomorphic covering of $X$. Let $G$ be the group of automorphisms of the strict equivariant covering $\tilde{p}$. Then,

1. the group $G$ is isomorphic to the strict fundamental group $\sigma_{1}(X, \Sigma)$ of $X$;

2. the group $G$ is a loxodmmic Kleinian subgroup of $\mathrm{PGL}_{2}(\mathbb{R})$ with $\Omega$ as region of discontinuity;

3. the quotient Riemann surface $\Omega / G$ is equivariantly isomorphic to $X$.

Moreover, the following equivalences hold.

4. The group $G$ is nonelementary if and only if $g \geq 2$.

5. The real Kleinian group $G$ is of the second kind if and only if $X$ has real points.

6. The group $G$ is Fuchsian if and only if $X$ is dividing.

Proof. Statement 1 is clear. Statement 3 follows from Proposition 2.5. To show statement 2 , it suffices to observe that the restriction of $\tilde{p}$ to the double half-plane $D$ is a universal equivariant covering of $X \backslash X^{\Sigma}$, and therefore, $G$ does not contain any elliptic or parabolic elements by Proposition 5.1. 
By Proposition 4.1, if $g=0$ or 1 then the group $G$ is commutative, in particular, elementary. If $g \geq 2$ then $G$ is not commutative. Since $G$ is loxodromic, $G$ is necessarily nonelementary. This shows equivalence 4 .

Since the equivariant covering $\tilde{p}$ is strict, $\tilde{p}^{-1}\left(X^{\Sigma}\right)=\Omega \cap \boldsymbol{P}^{\mathbf{l}}(\boldsymbol{R})$. Hence, $G$ is of the second kind if and only if $X^{\Sigma} \neq \emptyset$. This proves equivalence 5 .

Equivalence 6 is obvious.

Remark 6.2. Let $X$ be a compact connected real algebraic curve of genus $g$ having real points. Let $p: \Omega \rightarrow X$ be a strict uniformization of $X$ and let $G$ be the group equivariant automorphisms of the covering p. By Proposition $4.1, G$ is a Kleinian group freely generated by $g$ elements. Since any finitely generated free Kleinian group is a Schottky group [14], the group $G$ is a Schottky group. Sibner showed that a compact connected real algebraic curve can be uniformized by a classical Schottky subgroup of $\mathrm{PGL}_{2}(\mathcal{C})$ [16]. We will show in a forthcoming paper that the strict uniformization $p: \Omega \rightarrow X$ of $X$ is, in fact, a uniformization of $X$ by a real Schottky group [10].

\section{Global real analytic coordinates on real Teichmüller spaces}

In this section, a real or complex algebraic curve is understood to be compact and connected.

Let $X$ be a real algebraic curve. Let $T(X)$ be the complex Teichmüller space of $X$, i.e., its elements are pairs $(Y, f)$, where $Y$ is a complex algebraic curve and $f: X \rightarrow Y$ is an orientation-preserving quasiconformal homeomorphism. Two such pairs $(Y, f)$ and $(Z, h)$ represent the same element of $T(X)$ if and only if there is a biholomorphic map $k: Y \rightarrow Z$ such that $k \circ f$ is homotopic to $h$. It is known that $T(X)$ admits a natural structure of a complex analytic manifold. For that structure, $T(X)$ is connected and of dimension $3 g-3$ if $X$ is of genus $g \geq 2$ (see [15] for details).

The action of $\Sigma$ on $X$ induces an action of $\Sigma$ on $T(X)$. Indeed, one defines $\sigma \cdot(Y, f)$ as $\left(Y^{\sigma}, f^{\sigma}\right)$, where $Y^{\sigma}$ is the complex conjugate structure 
on the manifold $Y$ and $f^{\sigma}: X \rightarrow Y^{\sigma}$ is defined by $f^{\sigma}(x)=f(\sigma \cdot x)$. It is easily verified that this gives rise to an action of $\Sigma$ on $T(X)$. It turns out that $\sigma$ acts antiholomorphically on $T(X)$. Since $T(X)^{\Sigma}$ is nonempty, $T(X)^{\Sigma}$ is a real analytic manifold of dimension $3 g-3$ if $g \geq 2$. Furthermore, $T(X)^{\Sigma}$ is connected [4]. The real analytic manifold $T(X)^{\Sigma}$ is called the real Teichmüller space of $X$.

The real Teichmüller space of a real algebraic curve $X$ is of interest for the study of moduli of real algebraic curves having the same topological type as $X$ [7]. In this section we will construct a global system of real analytic coordinates on the real Teichmüller space of a real algebraic curve of genus $g \geq 2$.

Let $p: \Omega \rightarrow X$ be a strict uniformization of the real algebraic curve $X$. Let $G$ be the group of automorphisms of the equivariant covering $p$. Let $M(G)$ be the space of Beltrami coefficients for $G$ with support in $\Omega$ (see [11] for definitions or nontrivial unproved statements that appear without reference). Since $G$ is a subgroup of $\mathrm{PGL}_{2}(\mathbb{R})$, the group $\Sigma$ acts naturally on $M(G)$.

Recall that for any Beltrami coefficient $\mu \in M(G)$, there is a unique orientation-preserving quasiconformal selfhomeomorphism $w^{\mu}$ of $\boldsymbol{P}^{1}(\boldsymbol{Q})$ having 0,1 and $\infty$ as fixed points and which is such that its complex dilation is equal to $\mu$. Since the set of all orientation-preserving quasiconformal selfhomeomorphisms of $\boldsymbol{P}^{1}(\mathcal{Q})$ having 0,1 and $\underline{\infty}$ as fixed points is a group, one gets, by transport of structure, the structure of a group on $M(G)$. This structure on $M(G)$ is such that $\Sigma$ acts by homomorphisms.

Let $\mu$ be in $M(G)$. Then, for all $\alpha \in G$, the selfhomeomorphism $w^{\mu} \circ \alpha \circ\left(w^{\mu}\right)^{-1}$ of $\boldsymbol{P}^{1}(\boldsymbol{C})$ is a Möbius transformation. One defines a homomorphism of groups

$$
\iota^{\mu}: G \longrightarrow \mathrm{PGL}_{2}(\mathscr{C})
$$

by letting $\iota^{\mu}(\alpha)=w^{\mu} \circ \alpha \circ\left(w^{\mu}\right)^{-1}$ for any $\alpha \in G$. Of course, $\iota^{\mu}$ is an isomorphism of $G$ onto its image. The maps $\iota^{\mu}$ are called quasiconformal deformations of $G$. We put

$$
\operatorname{Def}(\mathrm{G})=\left\{\kappa: \mathrm{G} \rightarrow \mathrm{PGL}_{2}(\mathcal{Q}) \mid \exists \mu \in \mathrm{M}(\mathrm{G}): \iota^{\mu}=\kappa\right\}
$$

the set of quasiconformal deformations of $G$. Note that $\iota^{\mu}$ is equal to the inclusion of $G$ into $\mathrm{PGL}_{2}(\mathcal{C})$ when $\mu$ is the trivial Beltrami coefficient 0 . 
The action of $\Sigma$ on $\mathrm{PGL}_{2}(\mathcal{C})$ induces an action of $\Sigma$ on $\operatorname{Def}(\mathrm{G})$. The subset $\operatorname{Def}(G)^{\Sigma}$ of $\operatorname{Def}(G)$ is the subset of real quasiconformal deformations of $G$. Obviously, a quasiconformal deformation $\kappa: G \rightarrow \mathrm{PGL}_{2}(\mathcal{C})$ is real if and only if $\kappa(G) \subseteq \mathrm{PGL}_{2}(\boldsymbol{R})$.

One has a natural map

$$
\iota: M(G) \longrightarrow \operatorname{Def}(\mathrm{G})
$$

defined by letting the image of $\mu$ be $\iota^{\mu}$ for all $\mu \in M(G)$. This map is clearly equivariant. Let $M_{0}(G)$ be the subset of Beltrami coefficients $\mu$ in $M(G)$ such that the deformation $\iota^{\mu}$ is equal to the inclusion $\iota^{0}$ of $G$ in $\mathrm{PGL}_{2}(\mathcal{Q})$. Then, $M_{0}(G)$ is a subgroup of $M(G)$, stable for the action of $\Sigma$ on $M(G)$, and the map $\iota$ is a quotient map for the action of $M_{0}(G)$ on $M(G)$.

The set Def(G) of quasiconformal deformations of $G$ gets the structure of complex analytic manifold since $M_{0}(G)$ acts freely on $M(G)$. In fact, Def(G) is a connected complex analytic manifold of dimension $3 g-3$ if $g \geq 2$. The action of $\sigma$ on $\operatorname{Def}(G)$ is antiholomorphic. Since $\operatorname{Def}(G)^{\Sigma} \neq \bar{\emptyset}$, the set $\operatorname{Def}(G)^{\Sigma}$ of fixed points is a real analytic manifold of dimension $3 g-3$ if $g \geq 2$.

A useful fact on $M_{0}(G)$ is the following. An element $\mu \in M(G)$ belongs to $M_{0}(G)$ if and only if the restriction $w_{\mid \Lambda}^{\mu}$ of $w^{\mu}$ to the limit set $\Lambda$ of $G$ is equal to the identity.

It is clear that for $\mu \in M_{0}(G)$, the map $w^{\mu}$ maps the domain of discontinuity $\Omega$ into itself. One lets $\widetilde{M}_{0}(G)$ be the subset of Beltrami coefficients $\mu \in M_{0}(G)$ such that $w^{\mu}$, considered as a map from $\Omega$ into itself, is homotopic to the identity $\operatorname{map}_{\Omega}$ on $\Omega$. Then, $\widetilde{M}_{0}(G)$ is a normal subgroup of $M_{0}(G)$ which is stable for the action of $\Sigma$ on $M(G)$.

Let $T(G)$ be the quotient of $M(G)$ by the action of $\tilde{M}_{0}(G)$. Then, $T(G)$ has a natural structure of a complex analytic manifold and is called the Teichmüller space of $G$. In fact, $T(G)$ is a connected complex analytic manifold of dimension $3 g-3$ if $g \geq 2$. One has an induced action of $\Sigma$ on $T(G)$. Complex conjugation $\bar{\sigma}$ acts antiholomorphically on $T(G)$. It turns out that $T(G)$ is equivariantly biholomorphic to the complex Teichmüller space $T(X)$ of $X$. Let

$$
\varphi: T(X) \longrightarrow T(G)
$$

be such a biholomorphic map. 
The map $\iota: M(G) \rightarrow \operatorname{Def}(\mathrm{G})$ factorizes through the quotient map $M(G) \rightarrow T(G)$ and gives rise to an equivariant holomorphic map

$$
\pi: T(G) \longrightarrow \operatorname{Def}(\mathrm{G})
$$

In fact, $\pi$ is a universal holomorphic covering of $\operatorname{Def}(G)$. The group of automorphisms of this covering is equal to the quotient group $M_{0}(G) / \widetilde{M}_{0}(G)$.

Let Def $(G)^{\Sigma, 0}$ be the connected component of Def $(G)^{\Sigma}$ containing the inclusion $\iota^{0}: G \rightarrow \mathrm{PGL}_{2}(\mathcal{C})$.

Lemma 7.1. The induced map

$$
\pi^{\Sigma}: T(G)^{\Sigma} \longrightarrow \operatorname{Def}(\mathrm{G})^{\Sigma}
$$

maps $T(G)^{\Sigma}$ real bianalytically onto $\operatorname{Def}(G)^{\Sigma, 0}$.

Proof. Since $T(G)^{\Sigma}$ is connected and since $\pi(0)=\iota^{0}$, one has that the image $\pi\left(T(G)^{\Sigma}\right)$ of $T(G)^{\Sigma}$ is contained in $\operatorname{Def}(G)^{\Sigma, 0}$.

It is easy to see that $\pi^{\Sigma}$ is surjective onto $\operatorname{Def}(G)^{\Sigma, 0}$. Indeed, the restriction of $\pi$ to the inverse image $A=\pi^{-1}\left(\operatorname{Def}(G)^{\Sigma, 0}\right)$ is a covering of Def $(G)^{\Sigma, 0}$. Since $\Sigma$ acts trivially on the latter space, $\Sigma$ acts trivially on the connected component $C$ of $A$ that contains $T(G)^{\Sigma}$. Then,

$$
T(G)^{\Sigma} \subseteq C \subseteq A^{\Sigma} \subseteq T(G)^{\Sigma}
$$

Hence, $T(G)^{\Sigma}=C$ is a connected component of $A$. Therefore, $\pi^{\Sigma}$ maps $T(G)^{\Sigma}$ onto $\operatorname{Def}(\mathrm{G})^{\Sigma}$.

Let us show that $\pi^{\Sigma}$ is injective. Let $\mu$ and $\nu$ be Beltrami coefficients in $M(G)$ representing two elements of $T(G)^{\Sigma}$. Due to a result of Earle (see [4] or [7], Theorem 21.1), we may assume that $\mu$ and $\nu$ are in $M(G)^{\Sigma}$. Suppose that $\mu$ and $\nu$ are such that $\iota^{\mu}=\iota^{\nu}$. Then, $\omega=\mu \cdot \nu^{-1}$ is in $M_{0}(G)$. Hence, the map $w^{\omega}$ maps $\Omega$ into itself and is equal to the identity on the limit set $\Lambda$ of $G$.

Since $\mu$ and $\nu$ are in $M(G)^{\Sigma}, \omega$ is also in $M(G)^{\Sigma}$. Therefore, the map $w^{\omega}$ is equivariant. In particular, $w^{\omega}$ maps $\boldsymbol{P}^{1}(\boldsymbol{R})$ into itself. Since this map is the identity on $\Lambda \subseteq P^{1}(R)$, one has that any point $x$ of $\boldsymbol{P}^{1}(\boldsymbol{R})$ and its image $w^{\omega}(x)$ belong to the same connected component of $\boldsymbol{P}^{\mathbf{1}}(\boldsymbol{R}) \backslash \Lambda$. It follows that $w^{\omega}$, considered as a map from $\Omega$ into itself is homotopic to the identity. Hence, $\mu \cdot \nu^{-1}=\omega \in \widetilde{M}_{0}(G)$ and therefore, 
$\mu$ and $\nu$ give rise to the same element of $T(G)$. This shows that $\pi^{\Sigma}$ is injective.

Now that we have proved that $\pi^{\Sigma}$ is bijective onto $\operatorname{Def}(G)^{\Sigma, 0}$, it follows that $\pi^{\Sigma}$ is a real bianalytic map since its complexification $\pi$ is a holomorphic covering map.

We will now construct an equivariant holomorphic open embedding $\psi$ of $\operatorname{Def}(\mathrm{G})$ into $\mathbb{C}^{g g-3}$, in case that $X$ has real points and its genus satisfies $g \geq 2$.

According to Proposition 4.1, the group $G$ is freely generated by $g$ elements $\gamma_{1}, \ldots, \gamma_{g}$ of $G$.

Observe that the Möbius transformations $\gamma_{1}$ and $\gamma_{2}$ do not have fixed points in common. Indeed, if they had a common fixed point then they would have had both of their fixed points in common ([14], Proposition I.D.4). This would imply that there are nonzero integers $m$ and $n$ such that $\gamma_{1}^{m}=\gamma_{2}^{n}$. This contradicts the fact that $G$ is freely generated by $\gamma_{1}, \ldots, \gamma_{g}$.

Since $\gamma_{1}$ and $\gamma_{2}$ are loxodromic elements not having common fixed points, one may assume that $\gamma_{1}$ has 0 as attractive and $\infty$ as repelling fixed point and that $\gamma_{2}$ has 1 as attractive fixed point.

Define

$$
\psi: \operatorname{Def}(\mathrm{G}) \longrightarrow \boldsymbol{C}^{\mathrm{dg}-3}
$$

by letting

$$
\psi(\kappa)=\left(a_{3}, \ldots, a_{g}, b_{2}, \ldots, b_{g}, c_{1}, \ldots, c_{g}\right),
$$

where $a_{i}$ (resp. $b_{i}$ ) is the attractive (resp. repelling) fixed point of $\kappa\left(\gamma_{i}\right)$ and $c_{i},\left|c_{i}\right|<1$, is the multiplier of $\kappa\left(\gamma_{i}\right)$, for all $i=1, \ldots, g$.

Observe that $\psi$ is well defined into $\mathscr{C}^{g-3}$ since each Möbius transformation $\gamma_{i}$, for $i \geq 2$, is loxodromic and does not have $\infty$ as fixed point.

Proposition 7.2. Suppose that $X$ has real points and that its genus satisfies $g \geq 2$. Then, the map $\psi$ is an equivariant biholomorphic open embedding of $\operatorname{Def}(\mathrm{G})$ into $\mathbb{C}^{\partial g-3}$.

Proof. It follows from quasiconformal deformation theory that $\psi$ is a holomorphic map. It is clear that $\psi$ is equivariant. Since $\operatorname{dim} \operatorname{Def}(G)=$ $3 \mathrm{~g}-3$, it suffices to show that $\psi$ is injective in order to conclude that $\psi$ is an equivariant biholomorphic embedding. 
Suppose that $\kappa$ and $\lambda$ are in $\operatorname{Def}(G)$ such that $\psi(\kappa)=\psi(\lambda)$. By definition of quasiconformal deformations, $\kappa\left(\gamma_{1}\right)$ and $\lambda\left(\gamma_{1}\right)$ both have 0 as attractive and $\infty$ as repelling fixed point. Since their multipliers are equal, $\kappa\left(\gamma_{1}\right)=\lambda\left(\gamma_{1}\right)$. One similarly proves that $\kappa\left(\gamma_{2}\right)=\lambda\left(\gamma_{2}\right)$. It is obvious that $\kappa\left(\gamma_{i}\right)=\lambda\left(\gamma_{i}\right)$ for $i=3, \ldots, g$. Since $G$ is generated by $\gamma_{1}, \ldots, \gamma_{g}$, one has $\kappa=\lambda$. This proves that $\psi$ is injective.

Theorem 7.3. Suppose that $X$ has real points and that its genus satisfies $g \geq 2$. Let $\Psi: T(X) \rightarrow \mathbb{C}^{d g-3}$ be the map $\psi \circ \pi \circ \varphi$. Then, the induced map

$$
\Psi^{\Sigma}: T(X)^{\Sigma} \longrightarrow \mathbb{R}^{3 g-3}
$$

is a global system of real analytic coordinates on the real Teichmüller space $T(X)^{\Sigma}$ of $X$.

Proof. Of course, $\Psi^{\Sigma}=\psi^{\Sigma} \circ \pi^{\Sigma} \circ \varphi^{\Sigma}$. Now, $\varphi: T(X) \rightarrow T(G)$ is an equivariant biholomorphic map. Hence, $\varphi^{\Sigma}: T(X)^{\Sigma} \rightarrow T(G)^{\Sigma}$ is real bianalytic. By Lemma $7.1, \pi^{\Sigma}: T(G)^{\Sigma} \rightarrow \operatorname{Def}(G)^{\Sigma, 0}$ is real bianalytic. By Proposition $7.2, \psi: \operatorname{Def}(\mathrm{G}) \rightarrow \mathscr{C}^{\mathrm{Bg}-3}$ is an equivariant biholomorphic open embedding. Hence, $\psi^{\Sigma}: \operatorname{Def}(\mathrm{G})^{\Sigma} \rightarrow \mathbb{R}^{3 \mathrm{~g}-3}$ is a real bianalytic open embedding. Therefore, its restriction to the connected component $\operatorname{Def}(G)^{\Sigma, 0}$ is a real bianalytic open embedding too. It follows that $\Psi^{\Sigma}$ is a real bianalytic open embedding of $T(X)^{\Sigma}$ into $\mathbb{R}^{3 g-3}$, i.e., a global system of real analytic coordinates on $T(X)^{\Sigma}$.

The situation in the case of $X$ being a real algebraic curve without real points is rather different. In this case the global system of real analytic coordinates on $T(X)^{\Sigma}$ turns out to be the one induced by an equivariant global system of complex analytic coordinates on the entire complex Teichmüller space $T(X)$. The reason for this is that, since $X$ has no real points, a uniformization of $X$ as a real algebraic curve is necessarily a strict uniformization of $X$. Complex analytic coordinates on complex Teichmüller spaces obtained by quasiconformal deformations of the uniformization of a real algebraic curve $X$ have been constructed in the papers $[8,9]$.

For completeness, we treat briefly the construction of a global system of real analytic coordinates on $T(X)^{\Sigma}$ in the case that $X$ has no real points. It will then also be clear why in this case the coordinate system 
extends to an equivariant global system of complex analytic coordinates on $T(X)$.

Let $X$ be a real algebraic curve without real points. We suppose again that the genus of $X$ satisfies $g \geq 2$. By Proposition 4.1, there are elements $\gamma_{1}, \ldots, \gamma_{g+1}$ of $G$ satisfying the relation

$$
\gamma_{1}^{2} \cdots \gamma_{g+1}^{2}=1 \text {. }
$$

As before we may assume that $\gamma_{1}$ has 0 as attractive and $\infty$ as repelling fixed point and that $\gamma_{2}$ has 1 as attractive fixed point.

Then one defines a map

$$
\psi: \operatorname{Def}(\mathrm{G}) \longrightarrow \mathbb{C}^{\mathrm{dg}-3}
$$

by Equation 1, as in the case of $X$ having real points. Note that $\gamma_{g+1}$ does not intervene at all in the definition of $\psi$. Nevertheless, $\psi$ is injective. More precisely:

Proposition 7.4. Suppose that $X$ has no real points and that its genus satisfies $g \geq 2$. Then, the map $\psi$ is an equivariant biholomorphic open embedding of $\operatorname{Def}(\mathrm{G})$ into $\mathbb{C}^{3 g-3}$.

Proof. As before it suffices to show that $\psi$ is injective. But this is done in the proof of Theorem 5.2 of [8].

Theorem 7.5. Suppose that $X$ has no real points and that its genus satisfies $g \geq 2$. Let $\Psi: T(X) \rightarrow \mathscr{d}^{g-3}$ be the map $\psi \circ \pi \circ \varphi$. Then, $\Psi$ is an equivariant global system of complex analytic coordinates on the complex Teichmüller space $T(X)$ of $X$. In particular, the induced map

$$
\Psi^{\Sigma}: T(X)^{\Sigma} \longrightarrow R^{3 g-3}
$$

is a global system of real analytic coordinates on the real Teichmüller space $T(X)^{\Sigma}$ of $X$.

Proof. As before, $\varphi: T(X) \rightarrow T(G)$ is an equivariant biholomorphic map. Since $X$ does not have real points, the domain of discontinuity $\Omega$ of $G$ is equal to the double half-plane $D$. Since the connected components of $\Omega$ are simply connected, $\widetilde{M}_{0}(G)=M_{0}(G)$. It follows that the equivariant map $\pi: T(G) \rightarrow \operatorname{Def}(G)$ is biholomorphic. By Proposition 
7.4 , the map $\psi$ is an equivariant biholomorphic open embedding $\psi$ of $\operatorname{Def}(\mathrm{G})$ into $\mathbb{Q}^{8 g-3}$. Hence,

$$
\Psi=\psi \circ \pi \circ \varphi: T(X) \longrightarrow \mathscr{C}^{3 g-3}
$$

is an equivariant global system of complex analytic coordinates on the entire complex Teichmüller space $T(X)$.

Remark 7.6. It should be eminently clear that, in the case that $X$ has real points, the coordinate system $\Psi^{\Sigma}$ on the real Teichmüller space $T(X)^{\Sigma}$ of $X$ does not extend to a global coordinate system of complex analytic coordinates on the complex Teichmüller space $T(X)$ of $X$.

Acknowledgement. This paper has been written while I was staying at the Universidad Complutense de Madrid. I would like to thank heartily the Departamento de Álgebra and its staff-and in particular, Carlos Andradas-for the excellent conditions I enjoyed during my stay.

\section{References}

[1] Alling, N. L., Greenleaf, N., Foundations of the theory of Klein surfaces. Lect. Notes in Math. 219, Springer Verlag, 1971.

[2] Behnke, H., Sommer, F., Theorie de analytischen Funktionen einer komplexen Veränderlichen. Springer Verlag, Berlin, 1962.

[3] Bers, L., Correction to "Spaces of Riemann surfaces as bounded domains." Bull. Amer. Math. Soc. 67 (1961), 465-466.

[4] Earle, C. J., Moduli of surfaces with symmetries. Advances in the theory of Riemann surfaces. Ahlfors, L.V. et. al. (eds) Princeton University Press and University of Tokyo Press, 1971.

[5] Earle, C. J., Some intrinsic coordinates on Teichmüller space. Proc. Amer. Math. Soc. 83 (1981), 527-531.

[6] Farkas, H., Kra, I., Riemann surfaces, 2nd ed. Grad. Texts Math. Springer Verlag, Berlin, 1992.

[7] Huisman, J., Real Teichmüller spaces and moduli of real algebraic curves. (submitted). 
[8] Huisman, J., The equivariant fundamental group, uniformization of real algebraic curves, and global complex analytic coordinates on Teichmüller spaces I. (submitted).

[9] Huisman J., The equivariant fundamental group, uniformization of real algebraic curves, and global complex analytic coordinates on Teichmüller spaces II. (submitted).

[10] Huisman J., Schottky uniformization of real algebraic curves and an applications to moduli (submitted).

[11] Kra, I., Deformation spaces. A crash course on Kleinian groups. L. Bers, I. Kra (eds). LNM 400, Springer Verlag, Berlin, 1974, $48-70$.

[12] Lehto, O., Virtanen, K. I., Quasiconformal mappings in the plane. Springer Verlag, Berlin, Heidelberg, New York, 1973.

[13] Maskit, B., Moduli of marked Riemann surfaces. Bull. Amer. Math. Soc. 80 (1974), 773-777.

[14] Maskit, B., Kleinian groups. Springer Verlag, Berlin, 1988.

[15] Nag, S., The complex analytic theory of Teichmüller spaces. John Wiley \& Sons, 1988.

[16] Sibner, R. J., Uniformization of symmetric Riemann surfaces by Schottky groups. Trans. Am. Math. Soc. 116 (1965), 79-85.

Institut Mathématique de Rennes. Université de Rennes 1. Campus de Beaulieu, 35042 Rennes Cedex, France.

e-mail: buismancuniv-ronnes1.1r 